\title{
Android Application Based Bluetooth Controlled Robotic Car
}

\author{
Ayan Maity $^{1}$, Avijit Paul ${ }^{1}$, Priyanka Goswami ${ }^{2}$, Ankan Bhattacharya ${ }^{1}$ \\ ${ }^{1}$ Department of Electronics and Communication Engineering, Mallabhum Institute of Technology, Bishnupur, India \\ ${ }^{2}$ Department of Computer Science and Engineering, Guru Nanak Institute of Technology, Kolkata, India
}

\section{Email address:}

maityayan1996@gmail.com (A. Maity), avijit.pau106@gmail.com (A. Paul), Goswami.priyanka16@gmail.com (P. Goswami), bhattacharya.ankan1987@gmail.com (A. Bhattacharya)

\section{To cite this article:}

Ayan Maity, Avijit Paul, Priyanka Goswami, Ankan Bhattacharya. Android Application Based Bluetooth Controlled Robotic Car. International Journal of Intelligent Information Systems. Vol. 6, No. 5, 2017, pp. 62-66. doi: 10.11648/j.ijiis.20170605.12

Received: March 17, 2017; Accepted: April 14, 2017; Published: November 29, 2017

\begin{abstract}
We are now living in the $21^{\text {st }}$ century. Now, smart phone has become the most essential thing in our daily life. Android application based smart phones are becoming each time more powerful and equipped with several accessories that are useful for Robots. This project describes how to control a robot using mobile through Bluetooth communication, some features about Bluetooth technology, components of the mobile and robot. We present a review of robots controlled by mobile phone via moving the robot upward, backward, left and right side by the android application such as Arduino, Bluetooth. Bluetooth has changed how people use digital device at home or office, and has transferred traditional wired digital devices into wireless devices. Here we are using Bluetooth communication, interface microcontroller and android application. We are using Arduino software to interface the Bluetooth module with microcontroller. According to commands received from android the robot motion can be controlled. We derived simple solutions to provide a framework for building robots with very low cost but with high computation and sensing capabilities provided by the smart phone that is used as a control device.
\end{abstract}

Keywords: Wireless, Android, Bluetooth, Arduino, Robotic Car

\section{Introduction}

This paper represents android application based Bluetooth controlled robotic car. Here main motto of our project is to control the car with android application. Here we use mainly Arduino UNO (ATMEGA 328P), Bluetooth module (HC-05). We interface the Bluetooth module with the system so that we can easily control the system by smart phone application. This project is more necessary to the modern society in context of spying and surveillance. The project aims in designing a Robot that can be operated using Android mobile phone. The controlling of the Robot is done wirelessly through Android smart phone using the Bluetooth feature present in it. Here in the project the Android smart phone is used as a remote control for operating the Robot. The controlling device of the whole system is a Microcontroller. Bluetooth module, DC motors are interfaced to the Microcontroller. The data received by the Bluetooth module from Android smart phone is fed as input to the controller. The controller acts accordingly on the DC motors of the Robot. In achieving the task the controller is loaded with a program written using Embedded ' $\mathrm{C}$ ' language. Related reference articles implementing wireless control of robots have been studied as mentioned in [1-12]. Still there exists a requirement of a cost-effective automation system, which will be easy to implement. An example of such a costeffective project has been proposed here.

\section{System Design}

The design of the system is kept as simple as possible. Few things like cost-effectiveness and simplicity in design, lowprofile structure etc. have been kept in mind before designing the project. Our system aims to achieve the target to design a system that can provide following functionalities with a simple and easy-to-use interface:

a) Develop an android application that will act as an remote of a robot.

b) Develop a robot which will be helpful for travelling.

c) Here the focus is on the latest technology of android 
and robot also called as 'Mobot'.

d) An android smart-phone and the technology of android is vast and can be used to interact with embedded system.

e) Mobile, robot and Bluetooth are the on-going technologies which can be used for the benefit of mankind.

f) Hardware of this project consists of Arduino UNO, Bluetooth module and a motor driver IC.

g) The Bluetooth module is connected with the Arduino UNO board for the connection with the user.

h) Through the Bluetooth module for monitoring and controlling the particular motor reaches the board and process accordingly and the output of the Arduino goes to the motor driver IC and it controls the particular motor.

The system consists of following parts:

a) Arduino UNO (ATMEGA 328P)

b) Bluetooth module (HC-05)

c) Smart phone

d) Motor driver (1293D)

e) Arduino software (version 1.8.1)

The basic building blocks of the project have been described below:

\subsection{Arduino UNO}

Microcontroller will act as the brain of the robot. The robot movement will be decided by the microcontroller. In this system we will be using microcontroller named Arduino UNO which contains ATMEGA 328P microcontroller chip (Figure 1). The microcontroller is programmed with the help of the Embedded $\mathrm{C}$ programming. Arduino has it own programming burnt in its Read Only Memory (ROM). Cprogram is very easy to implement for programming the Arduino UNO.

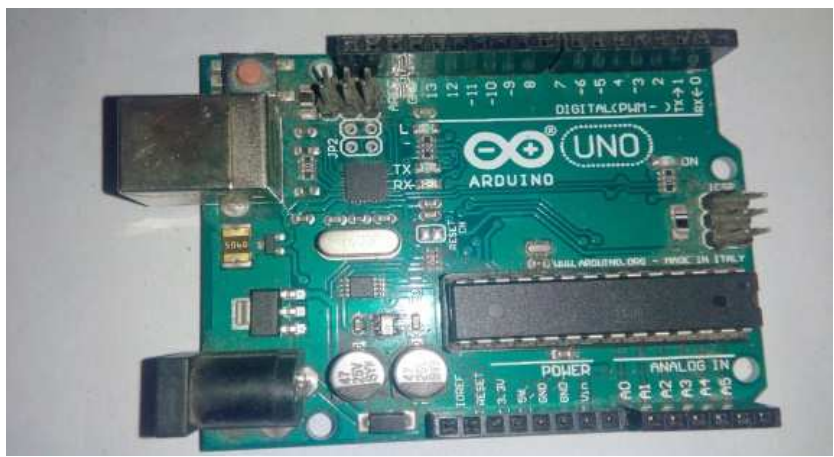

Figure 1. Arduino UNO

\subsection{Bluetooth Module (HC-05)}

The Bluetooth module will act as an interface between Smartphone and microcontroller. We will be using HC-05 Bluetooth module for the system, which can be used as either receiver or transmitter. Generally our transmitter will be smart-phone and receiver will be Bluetooth module (Figure 2). Bluetooth module will give the commands given by smart-phone to the microcontroller.

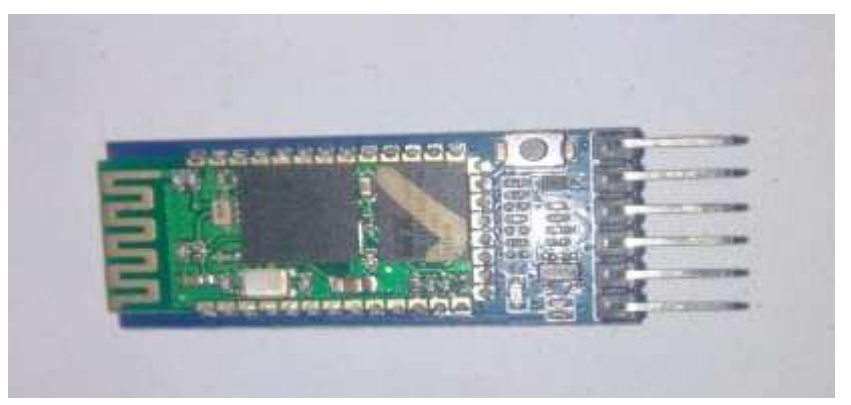

Figure 2. Bluetooth Module.

\subsection{Smart Phone}

The smart phone is the transmitter of this circuit. It sends the data to microcontroller through Bluetooth module. It also helps to send the instruction of forward, backward, left, right to the microcontroller. Actually, the smart phone is used as a remote of this system. Here we the Bluetooth RC Controller application (Figure 3 ) as the operating remote of this system. The advantage of this project is that the application software designed for android phones is kept simple but attractive with all necessary built-in functions. The novelty lies in the simplicity of the design and functioning.

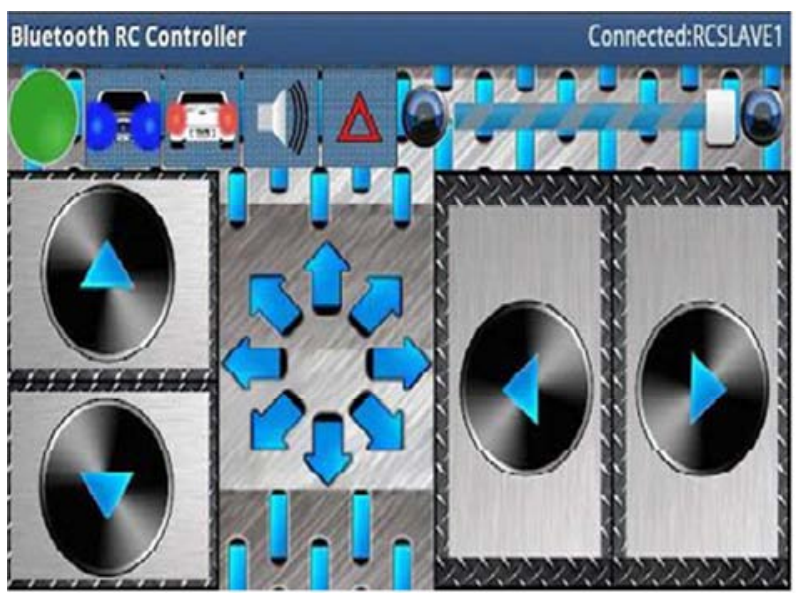

Figure 3. Smart Phone Application.

\subsection{Motor Driver (L293D)}

Motor driver IC is used to control the dc motors. It is also interfaced with the microcontroller (Figure 4 (a)) and with circuit connections (Figure 4 (b)).

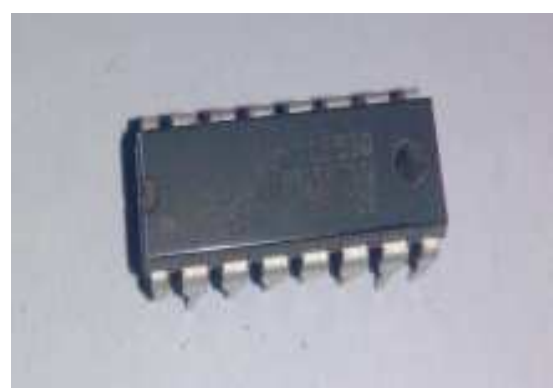

Figure 4a. Motor Driver IC. 


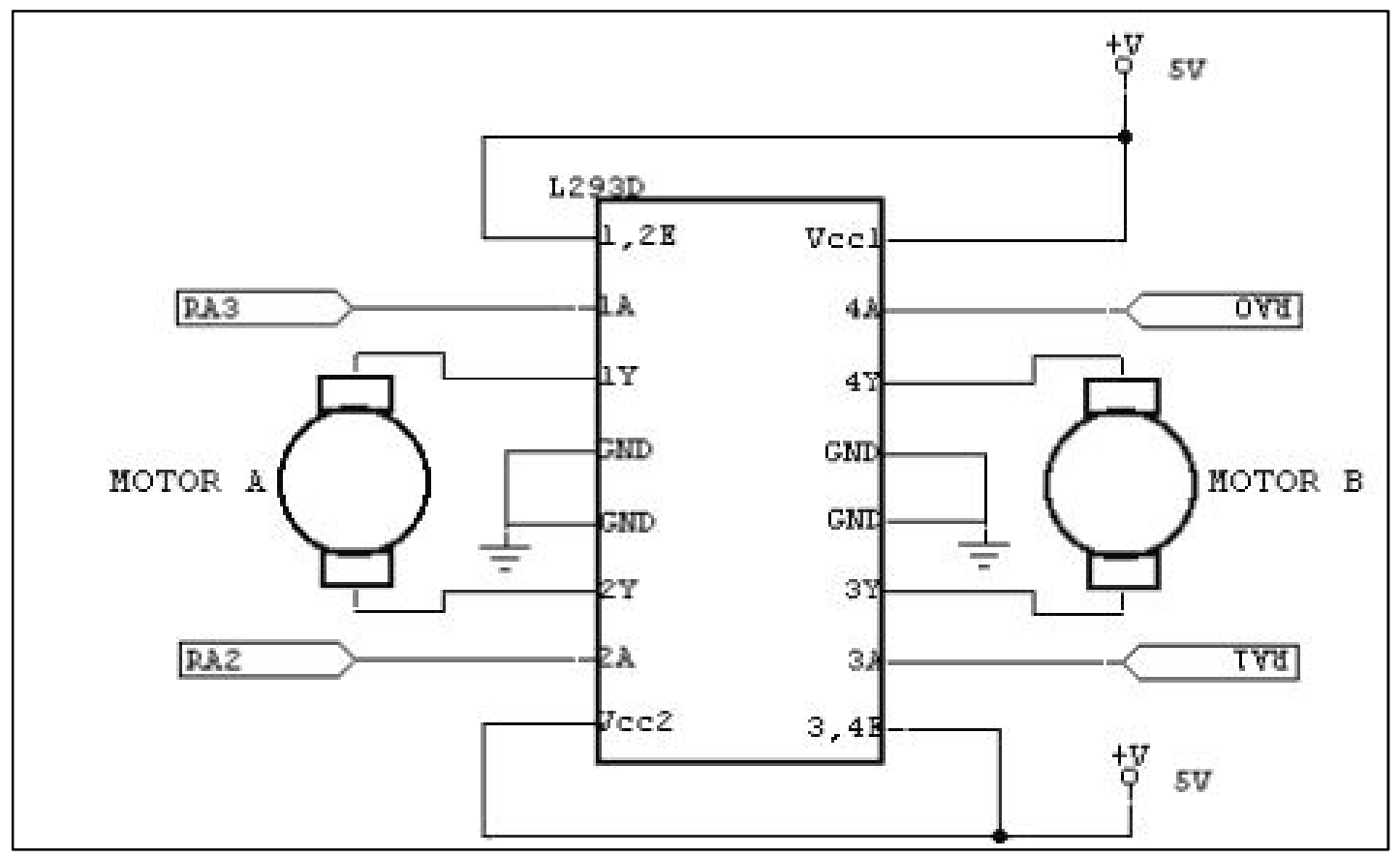

Figure 4b. Motor Driver IC with circuit connections.

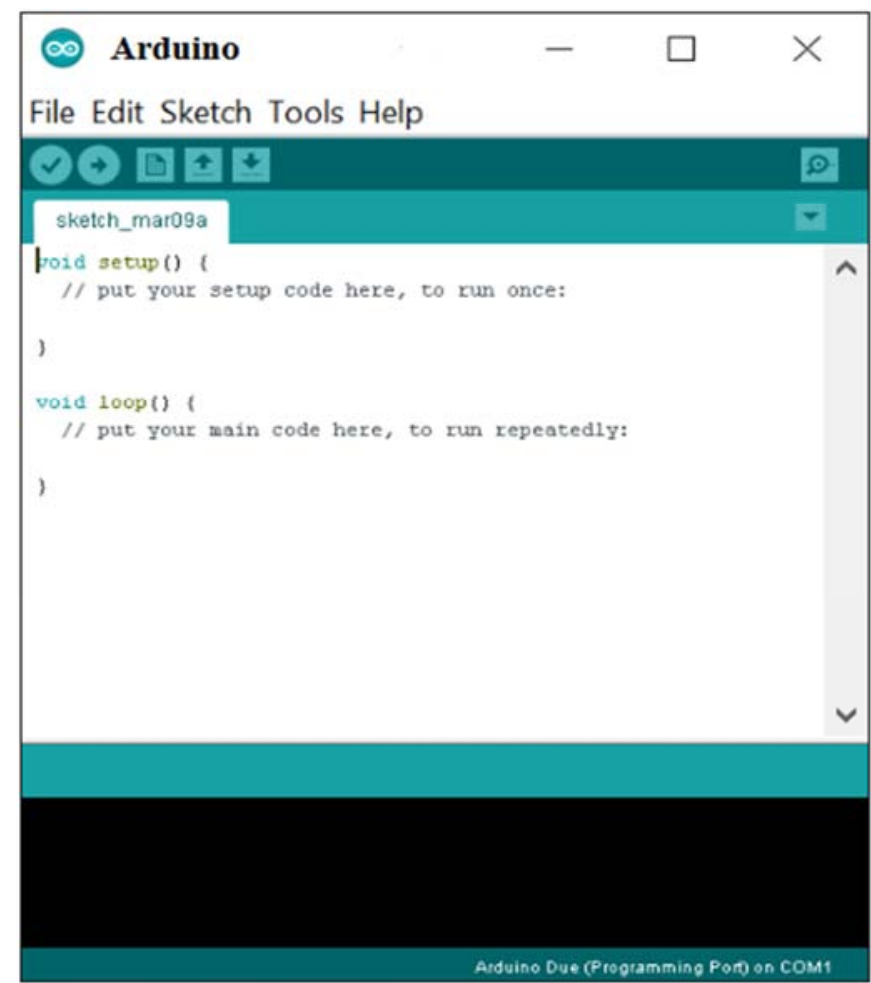

Figure 5. Arduino Software Interface.

\subsection{Arduino Software (Version 1.8.1)}

Arduino software (Figure 5) is used to put the instruction of whole functions of this system to the microcontroller. Here we use programming language ' $\mathrm{C}$ ' for coding. The program for executing this project has been written in $\mathrm{C}$ language. The program is burnt in the microcontroller using burner software. The program is stored in the EEPROM of the microcontroller, which is present in the Arduino board. By this software we put the data and instruction for forward, backward, left, right operation of this system.

In android application when we press a button, a corresponding signal is sent through the Bluetooth to Bluetooth module (HC-05) which is connected with the Arduino board. When signal data arrives the Arduino the pin which corresponds to the particular input is set to high. Now that pin gives the output to the motor driver section. Motor driver switches accordingly the data bit, if the data bit is low then the corresponding pin of the motor driver doesn't work else highbit then thecorrespondingpinof the motor driver is on. We have used Arduino IDE version 1.8.1 for writing program for Arduino. There are two steps of the programming. First set up section where we define all the variables. Second loop part where the program runs continuously.

\section{Working Principle}

The working principle is kept as simple as possible. The working principle of the circuit has been elaborated with the help of a block diagram, of the system interconnection as shown in Figure 6. As seen from the Figure 6. A DC power supply is required to run the system. The DC power supple feeds the Microcontroller and the Bluetooth module. The Bluetooth module receives the signal sent from an android smart-phone, where the application software coded in $\mathrm{C}$ language is installed. The microcontroller, thereby, sends instructions, which when executed, helps in functioning of the motor driver. The movement and functioning of the motor can be controlled by using the android based application software. 


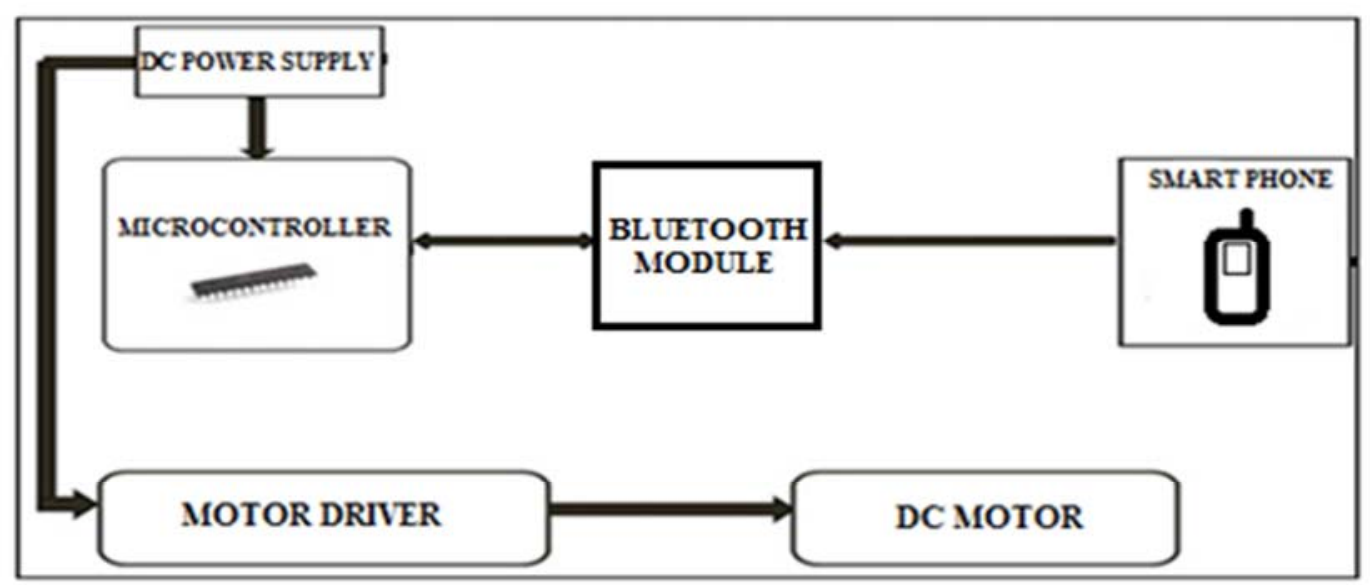

Figure 6. Block diagram of the proposed system.

Hardware of this project consists of Arduino UNO, Bluetooth module and a motor driver IC. The Bluetooth module is connected with the Arduino UNO board for the connection with the user. Through the Bluetooth module for monitoring and controlling the particular motor reaches the board and process accordingly and the output of the Arduino goes to the motor driver IC and it controls the particular motor.

Our proposed project consists of the following three sections:

a) Input section

b) Microcontroller section

c) Output section

In our android application base Bluetooth controlled robotic car, the user interacts with the system with a smart phone. In this method user must be present within in range ( $<$ 15 meters) to control the system. In future we would try to extend the range using Internet of Things (IoT) [12]. When user sends any data to the Arduino board then the corresponding pin of Arduino goes to high state and switches the motor driver ic in the on mode. The corresponding motor moves as per the input data. Here in this project the user (android application) is the input section. This device is connected with the Arduino board (microcontroller section) by the means wirelessly i.e. Bluetooth module. The system can now be connected with the motors (output section) to be controlled via wireless connectivity.

The circuit diagram of this project is shown below:

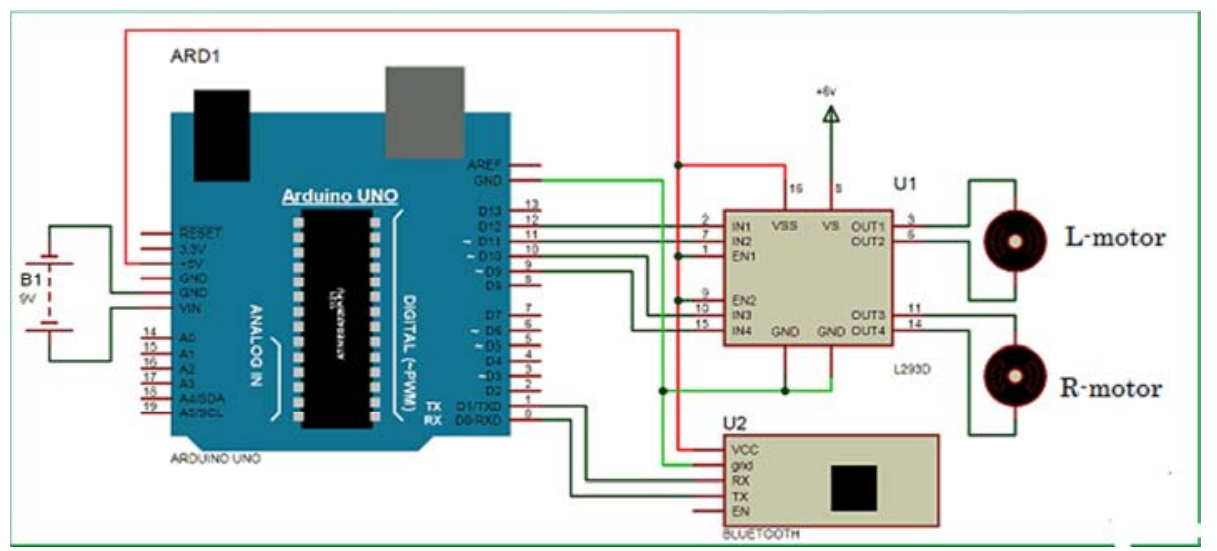

Figure 7. Circuit diagram of the project.

Here at first we construct the circuit as shown in Figure 7. Then through the data cable we insert the commands in the microcontroller ATMEGA 328P. These commands help the microcontroller to interface with the Bluetooth module HC05 and also with the motor driver IC L293D. Here the Bluetooth module act as a receiver which receives the instruction from the smart phone (remote or transmitter). Then the microcontroller decides the operation for the instruction which is coming from the smart phone. The functions of the given instructions are operated by the microcontroller. The instructions are sent by the smart phone. We can easily control the movements of the dc motor. The Bluetooth module can operate below the $10 \mathrm{~m}$ range, which we would try to extend in future. Here we are using four 12 V, 200 R.P.M DC motors and a 9 V DC battery as main power supply of this system. Until we send any instruction to the microcontroller the motors remain stop. When any input is given then the motors moves as per the preloaded functions in the microcontroller. Figure 8 shows the snapshot of the whole Bluetooth Based Smart Phone Control Robot Project. 


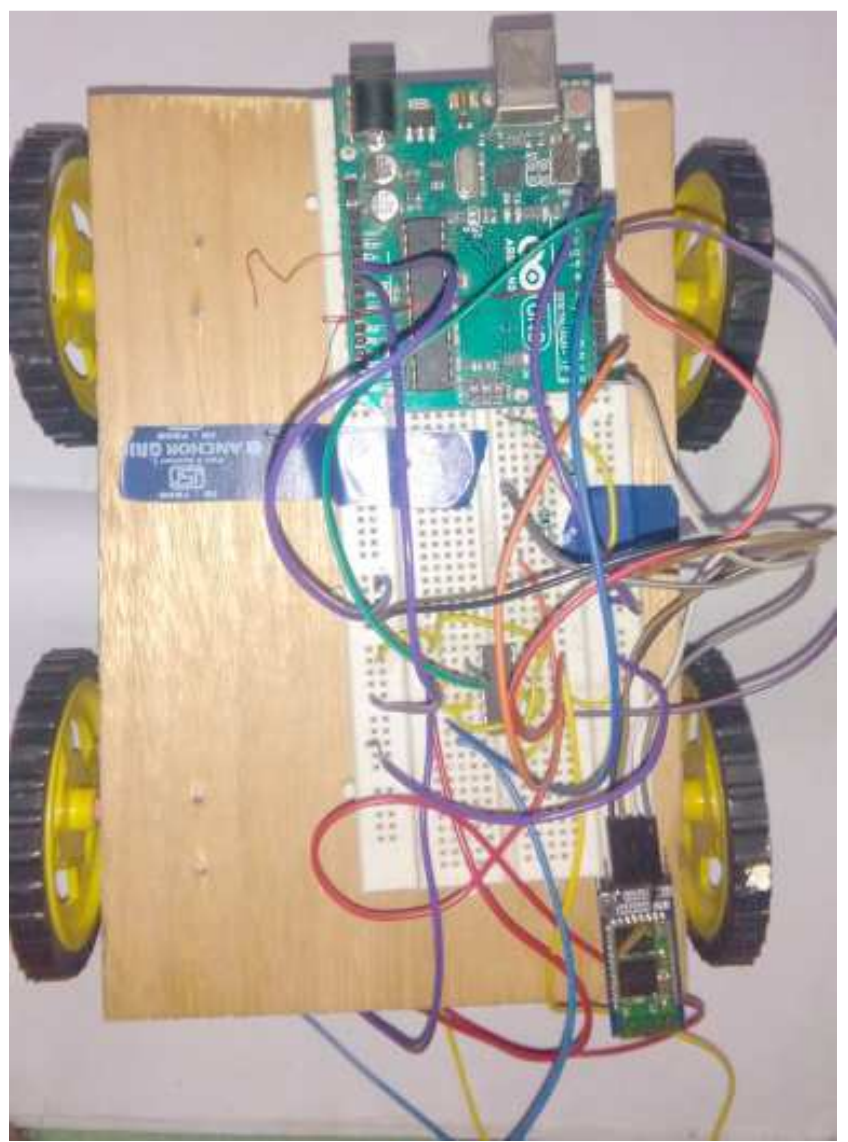

Figure 8. Snapshot of Bluetooth based Smart Phone Control Robot Project.

\section{Conclusion}

This is indeed a cost-effective and efficient project. The novelty lies in the fact that it is a cost-effective project with a simple and easy to use interface compared to existing ones. Also the Bluetooth RC Controller application is more user friendly. The robot is small in size so it can be used in spying purpose. With few additions and modifications, this robot can be used in army for detecting and disposing hidden land mines. The robot can be used for surveillance. In future we can interface sensors to this robot so that it can monitor some parameters and we can improve the efficiency using Internet of Things (IoT) technology. We can also add wireless camera, in order to incorporate other security features.

\section{References}

[1] Xiaoluet.al. "Robot control design based on smartphone." Control and Decision Conference (CCDC), $201325^{\text {th }}$ Chinese. IEEE, 2013.

[2] Yeon-Gyunkim et.al. "Smartphone-controlled user calling system for a mobile robot."Robotics (ISR), $201344^{\text {th }}$ International Symposium on. IEEE, 2013.

[3] Rouanet, Pierre, et.al. "The impact of human- robot interfaces on the learning of visual objects." Robotics, IEEE Transactions on 29.2 (2013): 525-541.

[4] Tatiana Alexenko et.al. "Android-based speech processing for eldercare robotics." Proceedings of the companionpublication of the 2013 international conference on intelligent user interfaces companion. ACM, 2013.

[5] M. Young, The Technical Writer's Handbook. Mill Valley, CA: University Science, 1989.

[6] https://developer.android.com/training/basics/firstapp/index.ht $\mathrm{ml}$

[7] https://www.codeproject.com/Articles/628894/Learn-How-toDevelop-Android-Application

[8] https://www.tutorialspoint.com/android/

[9] http://www.coreservlets.com/android-tutorial/

[10] Arpit Sharma, ReeteshVerma, Saurabh Gupta and Sukhdeep Kaur Bhatia, "Android Phone Controlled Robot Using Bluetooth", International Journal of Electronic and Electrical Engineering. Volume 7, Number 5 (2014), pp. 443-448

[11] A. Sharma, J. Mondal, C. Pandey, R. Kumar and A. Bhattacharya, "IoT Based Home Automation System"; Advances in Applied Science; Volume 2017; Article ID 100005, pp. 01-06; Feb. 2017

[12] A. Sharma, J. Mondal, C. Pandey, R. Kumar and A. Bhattacharya, "IoT Based Home Automation System Using Arduino"; $7^{\text {th }}$ All India Inter Engineering College Academic Meet - 2016\& Innovative Model Competition for a Sustainable Society on 13th March, 2016; Organized by Forum of Scientists, Engineers \& Technologists (FOSET) in association with Supreme Knowledge Foundation Group of Institutions, India. 